Revue Française de Civilisation Britannique

XXI-2 | 2016

Economic Crisis in the United Kingdom Today: Causes and Consequences

\title{
En guise de conclusion
}

By Way of Conclusion

Catherine Marshall

\section{OpenEdition}

1 Journals

Édition électronique

URL : http://journals.openedition.org/rfcb/1119

DOI : $10.4000 /$ rfcb. 1119

ISSN : 2429-4373

Éditeur

CRECIB - Centre de recherche et d'études en civilisation britannique

Référence électronique

Catherine Marshall, «En guise de conclusion », Revue Française de Civilisation Britannique [En ligne], XXI-2 | 2016, mis en ligne le 05 octobre 2016, consulté le 19 avril 2019. URL : http://

journals.openedition.org/rfcb/1119; DOI : 10.4000/rfcb.1119

Ce document a été généré automatiquement le 19 avril 2019

\section{(c) $(1) \odot$}

Revue française de civilisation britannique est mis à disposition selon les termes de la licence Creative Commons Attribution - Pas d'Utilisation Commerciale - Pas de Modification 4.0 International. 


\title{
En guise de conclusion
}

\author{
By Way of Conclusion
}

\section{Catherine Marshall}

1 Comment imaginer qu'il y ait un sens à conclure ce numéro sur la crise ? Comment, alors que le terme revient sans cesse et que la notion prend de nombreux sens, imaginer y apporter une réponse finale ? Pourtant, à bien des égards, ce numéro consacré à la crise au Royaume-Uni apporte des réponses nouvelles à la situation outre-Manche des années 1960 à nos jours et force à modifier notre point de vue sur les conséquences de l'après 2008.

2 En effet, ce que les articles publiés dans cette revue tendent tous à montrer est bien que la notion de crise est féconde et qu'elle peut se percevoir comme un élément bien plus positif qu'on ne pourrait le penser au premier abord. En d'autres termes, il n'y a pas de théories adéquates pour décrire ce qu'est une crise : elle est généralement un signal, voire une force qui secoue pour aller de l'avant, mais elle s'inscrit dans une histoire qu'il faut disséquer pour la comprendre - dans le cas présent, celle du Royaume-Uni dans les cinquante dernières années ou bien davantage encore, comme le donne à voir l'article de notre collègue Simon Lee.

3 Ainsi, s'il n'y a pas de sens à conclure sur la notion de crise en général, il reste néanmoins à terminer sur une note plus optimiste, comme Stéphane Guy l'a esquissée à la fin de son introduction. Une grande partie des articles de ce numéro montrent que cette notion renferme les clés pour imaginer une sortie ou pour accepter de vivre avec la crise car elle fait partie de notre vie. L'issue passe par un réveil parfois brutal, un bilan d'une situation pénible et par la prise de décisions pour tenter d'avancer. Il invite à une transformation et parfois, il s'agit bien, d'une progression : d'un changement pour le meilleur.

4 De la crise financière - dans toute la réalité de ses conséquences sociales ici particulièrement étudiées par Ondine Aza et Raphaelle Espiet-Kilty -, à la crise politique, en passant par l'usage fait de cette notion, il apparait clairement qu'elle remet en question les certitudes et force à s'adapter. C'est sans doute pour cette raison, parce qu'elle touche à la fois plusieurs niveaux de notre conscience, qu'elle reste encore une notion à défricher et ce, tout particulièrement, par les civilisationnistes. 
5 Dans les cinq dernières années, la communauté scientifique des anglicistes s'y est fortement intéressée. Déjà en 2012, la revue Cercles avait consacré son $25^{\text {ème }}$ numéro, sous la direction de Pierre Sicard, à « La crise ». Plusieurs de nos collègues anglicistes, à la fois littéraires et civilisationnistes, étaient revenus sur « La crise dans sa dimension de remise en cause des certitudes individuelles et collectives; la crise par les bouleversements qu'elle induit dans l'écoulement habituel du temps $»^{1}$. En 2015, c'est l'équipe de nos collègues irlandistes, sous la direction de Martine Pelletier et de Valérie Peyronel, qui avait consacré un numéro d'Etudes irlandaises à «La crise? Quelle Crise? " ${ }^{2}$. Cette fois, l'idée était bien de chercher à distinguer à quelles crises il était fait référence et du bon usage du terme pour en éviter les travers dans le cadre des études irlandaises.

Dans le cas du présent numéro, l'accent a été mis sur deux versants de cette notion au Royaume-Uni : d'une part, la réalité des crises en politique et l'impact de 2008 ; d'autre part, l'usage que les hommes politiques ont pu en faire. Ce que les articles tendent à montrer est bien que si crise(s) il y a, il n'en demeure pas moins que le danger reste la réponse appropriée et son utilisation politique. Au lieu de profiter du signal de la crise pour faire une mise au point et chercher une issue, cette dernière est souvent utilisée politiquement pour subvertir le signal initial. C'est en cela que la crise finit par devenir permanente, comme une rengaine dangereuse, qui empêche de bouger et de trouver une issue. La notion perd tout son sens et son utilité est renversée, souvent au profit d'une idéologie. Anxiogène et perverse, elle sert de bouc-émissaire et ne joue plus son rôle positif. Pire encore, elle en vient à provoquer l'apathie des citoyens.

7 Ainsi, au-delà du premier signal, la crise devrait être aussi comprise comme un avertissement à se méfier de l'usage qui en sera fait - généralement politiquement. Il y a comme une injonction faite à tous les citoyens que nous sommes à nous responsabiliser pour ne pas accepter l'idée de crise comme une fatalité mais à en saisir toutes les opportunités. C'est dans cette responsabilisation de l'individu que réside la liberté de décider de la réponse à donner.

Dans le présent numéro, l'ambiguïté sémantique de cette idée prend tout son sens puisque sans fin, depuis les années 1960, le terme est utilisé pour pousser à des changements politiques, économiques et sociaux qui imposeraient une culpabilisation des citoyens et permettrait de réformer l'intervention publique induite par les théories keynesiennes de la croissance (et sa lutte contre le chômage en particulier) depuis la fin de la seconde guerre mondiale. La grande période de difficultés traversée par le Royaume-Uni tout au long des années 1970 - de la crise du pétrole en 1973, au Winter of Discontent de 1978-1979, en passant par la dévaluation de la Livre et le recours à l'aide du FMI en 1976 - n'aurait été qu'une longue série d'épreuves dont Margaret Thatcher aurait compris la portée et saisi l'opportunité de réformer l'Etat au profit d'une idéologie néoconservatrice fondée sur le libéralisme de marché. Le gouvernement Cameron-Clegg de 2010 à 2015 n'aurait pas fait mieux que les gouvernements successifs de Margaret Thatcher de 1979 à 1990, en se servant des conséquences de 2007-2008 pour imposer d'avantage d'austérité fiscale.

9 Pourtant, et l'article de Simon Lee est particulièrement éclairant à ce titre en particulier, il faut replacer les réponses politiques aux crises économiques des quarante dernières années outre-Manche dans un contexte quelque peu différent de celui généralement présenté, à savoir uniquement celui de la mise en place du néo-libéralisme après ce qui a été trop rapidement qualifiée de période de « consensus politique » de 1945 à $1975 .{ }^{3}$ Le rôle de l'Etat britannique dans sa réponse aux différentes crises politico-sociales depuis 
les années 1970 a aussi été de redéfinir son propre rôle en faisant en sorte de défendre ses intérêts face au pouvoir financier et monétaire. Affirmer cela n'est pas dire que l'Etat l'a fait au profit des citoyens mais bien qu'il faut davantage se pencher sur les réponses données aux vagues successives de crises financières depuis les années 1970 dans un temps plus long pour en mesurer toute la portée. En ce sens, l'Etat britannique ne réagirait, comme le pense Simon Lee, que de la façon dont il a toujours réagi depuis la fin $\mathrm{du} 18^{\mathrm{e}}$ siècle et qu'il serait réducteur de ne pas remettre les crises successives que peuvent rencontrer les pays dans leur contexte historique et surtout politique.

En 1938 déjà - mais dans un autre sens que celui présenté par Simon Lee -, dans Parliamentary Government in England, le constitutionnaliste Fabien Harold Laski, dénonçait la crise des années trente comme étant le résultat d'un système politique britannique dans lequel s'opposaient démocratie et capitalisme. A ses yeux, la Glorieuse Révolution de 1688 avait été faite au nom de la liberté individuelle et de la protection de la propriété privée. Ainsi, le régime parlementaire britannique qui en avait découlé était-il fondé sur des idéaux - la liberté et l'individualisme - qui défendaient un capitalisme dérégulé. Comment, alors que le Royaume-Uni était devenu une véritable démocratie en 1918 (mais véritablement en 1928 avec le droit de vote donné pratiquement à tous les hommes et femmes de plus de 21 ans) et que l'Etat s'employait à prendre en compte les besoins les plus importants des citoyens, concilier ce qui relevait de la liberté et ce qui relevait de l'égalité ? Aux yeux de Laski, il existait une contradiction interne au système, opposé aux demandes de la population dans les années 30. "Our society", écrivait-il,

is overwhelmingly [...] an acquisitive society, and its main governmental apparatus is in the hands of those who have been themselves successful in acquisition. It is they who determine what the State shall do with its power. It is they who examine the needs of our society and decide to which of those needs, and in what degree, response shall be made ${ }^{4}$.

11 Dans un tel système, l'Etat défenseur de l'individualisme et de la propriété privée mettait en scène son rôle en apprenant l'obéissance et la déference des citoyens envers le régime politique - la remise en cause du système était alors impossible. Or, nous dit Laski, c'était bien le système politique lui-même, plutôt que la crise financière des années 1920, qui était responsable de crises et difficultés en cours dans ces années. Au début du vingtième siècle, poussé par certains hommes politiques, tel le Libéral David Lloyd George, issus de milieux simples qui s'étaient hissés au pouvoir grâce à l'extension du droit de vote, le régime avait commencé à répondre aux besoins des citoyens et à jeter les bases de ce qui deviendra après 1945 l'Etat-providence mais, dans les années 1930, le système montrait déjà des limites évidentes. Entre démocratie et capitalisme dérégulé, il faudrait choisir.

Harold Laski, qui écrivait à la veille de la seconde guerre mondiale, voyait néanmoins certaines issues, en particulier, il gardait foi en la capacité du système parlementaire à se transformer et à évoluer face à une nouvelle crise. Il rappelait que les changements du dix-septième siècle avaient résulté de grandes périodes d'instabilité et de crises majeures qui avaient imposé des changements profonds au système et un lien nouveau entre gouvernants et gouvernés. A l'instar de ce qui s'était passé en 1688, le temps était venu à la fin des années 1930, pensait Laski, de reconsidérer les fondements capitalistes du gouvernement parlementaire sans quoi le pays courrait le risque d'une rupture profonde au sein de la société. Il concluait ainsi en guise d'avertissement: " We can only make the adjustement our situation requires in the degree to which we are conscious of the dangers we confront. For it is, above all in our awareness of peril that we seem to hear the call to act while there is still time $»^{5}$. Finalement, c'est le second conflit mondial qui vint 
répondre à une grave crise financière et sociale par une guerre. Si, après 1945 , le gouvernement travailliste d'Attlee avait bien entendu cet appel et donné un nouveau souffle au rôle de l'Etat en le faisant le protecteur des citoyens, le système keynesien montra ses limites comme l'expliquent de nombreux articles présentés dans cette revue. Laski avait-il vu juste en identifiant que seule une transformation des fondements du régime parlementaire, en inscrivant dans le droit la protection des citoyens face à un capitalisme forcené serait la seule réponse adaptée? Ne serait-ce pas là une transformation radicale, contraire aux usages britanniques, identique à une petite révolution?

13 Harold Laski opérait aussi un glissement intéressant en ce sens en montrant que le réponse à une crise économique passait par une compréhension du rôle de l'Etat et, par extension, pour toute personne qui s'intéresse au Royaume-Uni, au fait de bien discerner les sources de la Constitution britannique non-codifiée. Laski écrivait à une période où la flexibilité de la Constitution était encore célébrée et où son caractère historique en faisait sa force. Plusieurs changements de taille ont eu lieu de nos jours concernant la Constitution britannique, du transfert de pouvoirs spécifiques aux régions du Royaume avec la dévolution à la fin des années 1990 au Constitutional Reform Act de 2005. Cette dernière loi donna un coup d'arrêt à la notion de «fusion » des trois grands pouvoirs, interprétés par Walter Bagehot dans son ouvrage The English Constitution (1867), pour garantir l'indépendance du pouvoir judiciaire et l'idée de séparation des pouvoirs (même si exécutif et législatif sont encore liés par le fait même que les membres du Cabinet sont membres de la Chambre des Communes et que le monarque demeure, quant à lui, la pierre angulaire du système). La loi de 2005 modifia considérablement le rôle du Lord Chancellor, donna lieu à la création d'une Cour Suprême et à un département des affaires constitutionnelles, qui a aujourd'hui rejoint le Ministère de la Justice. Pour la première fois également, une référence à la rule of law (la suprémacie du droit) était inscrite dans la loi. La version simplifiée que le constitutionnaliste A. V. Dicey donnait de la rule of law à la fin du dix-neuvième siècle, à savoir une absence de pouvoir arbitraire, le fait que nul ne soit au-dessus des lois et que le droit soit interprété par les juges au cas par cas, était habile et expliquait le caractère singulier de la Constitution. ${ }^{6}$ Il s'agissait d'une description adaptée au contexte victorien dans tout ce qu'elle avait d'anglo-centré et de conservateur.

14 Aujourd'hui, alors que le Royaume-Uni a bien changé, la reconnaissance des droits fondamentaux est à l'ordre du jour. L'ouvrage rédigé par l'ancien Lord Chief Justice, Tom Bingham, intitulé simplement The Rule of Law (2010), est instructif à plus d'un titre qui donne une définition bien plus étendue de ce principe fondateur de la Constitution suite, précisément, au Constitutional Reform Act de 2005. Cet essai montre bien que l'évolution de la Constitution va vers une reconnaissance et une formalisation codifiée des droits fondamentaux des britanniques pour les protéger du pouvoir parfois arbitraire de l'Etat. ${ }^{7}$ Ces changements en cours, qui passent aussi par la possibilité de comprendre les conventions non écrites de la Constitution - la rédaction d'un manuel à l'usage des membres du Cabinet ${ }^{8}$ va également dans ce sens - visent à rendre plus lisibles un système qui ne l'était pas. La Constitution non codifiée est devenue une singularité qui empêche la participation pleine et entière des citoyens à la politique du pays. Pour reprendre les mots du constitutionnaliste Vernon Bogdanor en 2015, «A codified constitution could help make Britain a genuine home for all its citizens "». Dans le monde présent non seulement soumis aux crises économiques mais aussi (ce qui est souvent lié) à de 
nouvelles crises, de type terroriste par exemple, cet avertissement n'est pas à prendre avec légèreté. Les crises vécues de nos jours ne justifient pas nécessairement que les libertés civiles soient mises en danger et que le pouvoir exécutif devienne arbitraire sous couvert de défendre la sécurité des citoyens. Harold Laski,, il y a presque 80 ans, pointait du doigt ce qui est compris de nos jours, à savoir que les crises économiques sont en partie une résultante de situations politiques et c'est sans doute aux citoyens d'en prendre davantage conscience, pour prendre réellement part aux changements possibles et de se défendre sur le plan politique, économique et social.

Ainsi, si les crises ne peuvent être appréhendées que dans un temps assez long pour en comprendre l'enchaînement, il faut pourtant s'extraire de la crise afin de pourvoir réellement l'analyser et c'est bien tout le problème qu'ont rencontré nos collègues pour tenter de tirer les enseignements de 2008 alors même que les conséquences s'en font encore sentir. Car existe-t-il réellement un moment sans crises? Nos collègues, tout en prenant en compte cette aporie, parviennent à porter des éclairages utiles sur des évènements encore en cours, tout en nous aidant à les placer dans une histoire plus globale.

On y revient de nouveau, l'autre aspect qui apparait à la lecture des articles du numéro et qui devrait sans cesse nous maintenir en alerte, est bien une utilisation très adroite de cette notion par les gouvernements successifs, et pas uniquement depuis 2008, pour mettre en œuvre des politiques d'austérité, alors que la question se pose de savoir si la réponse politique à la crise économique et sociale est bien d'imposer davantage de rigueur. A trop vouloir utiliser le terme à mauvais escient et à tout va, les gouvernements jouent souvent à une forme de manipulation de l'opinion publique à des fins électorales. Comme le montre Edwige Camp dans son article sur les effets de la crise financière sur les questions constitutionnelles écossaises : une fois élu sur un programme anti-austérité, le SNP est forcé de tenir ses promesses électorales dans un contexte financier qui ne le permet pas.

17 Alors que penser? Que la coalition conservative/libérale-démocrate de Cameron-Clegg de 2010 à 2015, comme les gouvernements conservateurs de Margaret Thatcher de 1979 à 1990, n'avaient pas d'autre choix que celui de la rigueur budgétaire et qu'ils avaient au moins le mérite de tenir un discours austère mais cohérent? N'y a-t-il pas un autre chemin à emprunter dans lequel les citoyens seraient les acteurs du changement au lieu de celui d'une finalité politique fondée sur une idéologie néolibérale? L'essai de James Connelly sur la façon de raisonner à travers les crises apporte un début de réponse, qui montre que toute forme de crise peut être pensée dans la perspective d'une progression.

Dans ce sens, les crises successives que le Royaume-Uni a rencontrées depuis les années 1970 détiennent en elles les germes de solutions à venir. Certains pourraient dire que les solutions tardent à émerger, mais il ne fait pas de doute que le chaos financier de 2007-2008, tout comme la période 1975-1976 au Royaume-Uni, permettent aux chercheurs que nous sommes de questionner les décisions politiques prises, de dénoncer l'utilisation parfois perverse du vocabulaire utilisé et de faire bon usage de la notion d'avertissement que véhicule n'importe quelle crise. Fondamentalement, il n'y a pas de période sans crises ; la seule distinction valable serait de séparer crises majeures - telles les guerres et les épisodes aux répercussions mondiales - et crises mineures - généralement nationales - mais, de nos jours, dans un monde globalisé, les liens entre les citoyens et les Etats sont tels qu'il serait sans doute judicieux d'accepter que tout relie les hommes du monde présent. Il faut y voir la possibilité de trouver des solutions globales, de ne pas être passifs 
ou ingénus face aux décisions politiques prises et de concevoir un monde moins enclin à défendre les disparités de biens entre les personnes. Pas à pas, ces crises politiques et financières sont une occasion de nous éduquer à une citoyenneté plus affirmée.

En ce sens, ces périodes difficiles sont riches de leçons pour les chercheurs et c'est aussi par les analyses et les études faites sur ces moments pénibles que viendront une partie des solutions. Ce sont ces articles, les échanges auxquels ils ont donné lieu, la possibilité de confronter les points de vue et les désaccords - en un mot, l'espace de liberté encore vivant qui réside dans nos universités en France - qui donnent un sens véritable à ce que nous sommes, à savoir des enseignants-chercheurs dont la recherche a pour mission de mieux comprendre le monde présent, de le questionner pour mieux y vivre, surtout et avant tout, en nos propres temps de crise.

Catherine Marshall est Maître de Conférences à Sciences Po Saint-Germain-en-Laye et membre du laboratoire AGORA. Ses recherches portent sur l'histoire des idées et la pensée politique du dix-neuvième siècle à nos jours. Elle a notamment publié plusieurs articles en histoire des idées, dont « The debate on vivisection within the Metaphysical Society " dans la RFCB, vol. XIX, n³. Elle a co-dirigé avec Bernard Lightman et Richard England, The Papers of the Metaphysical Society (1869-1880). A Critical Edition (OUP, 2015) et codirigé avec Stéphane Guy, The Victorian Legacy in Political Thought (Peter Lang, 2014).

\section{NOTES}

1. Pierre Sicard, «Introduction» dans: Cercles 25 (2012), pp. 2-3. www.cercles.com/n25/ introduction.pdf consulté le 30 avril 2016.

2. «La crise? Quelle crise?", Etudes irlandaises, 40-2 | 2015 Le numéro sera mis en ligne le 17 décembre 2017 : http://etudesirlandaises.revues.org/4728 consulté le 19 mai 2016.

3. Voir: Ben Pimlott, Dennis Kavabagh \& Peter Morris, "Is the "Postwar Consensus' a myth?", Contemporary Record, 2:6, pp. 12-5.

4. Harold Laski, Parliamentary Government in England (1938) (London, George Allen\& Unwin Ltd, 1945), p. 40.

5. Ibid., p. 70.

6. A. V. Dicey, Introduction to the Study of the Law of the Constitution (1885) (Indianapolis, Liberty Fund, $1885,8^{\text {th }}$ edition 1914).

7. Tom Bingham, The Rule of Law (London, Penguin Global, 2010), p. 208.

8. The Cabinet Manual. A guide to laws, conventions and rules on the operation of government (2011). Voir : https://www.gov.uk/government/uploads/system/uploads/attachment_data/file/60641/ cabinet-manual.pdf consulté le 10 aout 2016.

9. Vernon Bogdanor, "Magna Carta: The competing forces that cry out for a constitutional convention", The Independent, 14 June 2015. Voir : http://www.independent.co.uk/news/uk/ politics/magna-carta-the-competing-forces-that-cry-out-for-a-constitutionalconvention-10319520.html consulté le 7 août 2016. 


\section{AUTEUR}

CATHERINE MARSHALL

Sciences Po Saint-Germain-en-Laye 